\title{
Physiotherapy support for self-management of persisting musculoskeletal pain disorders
}

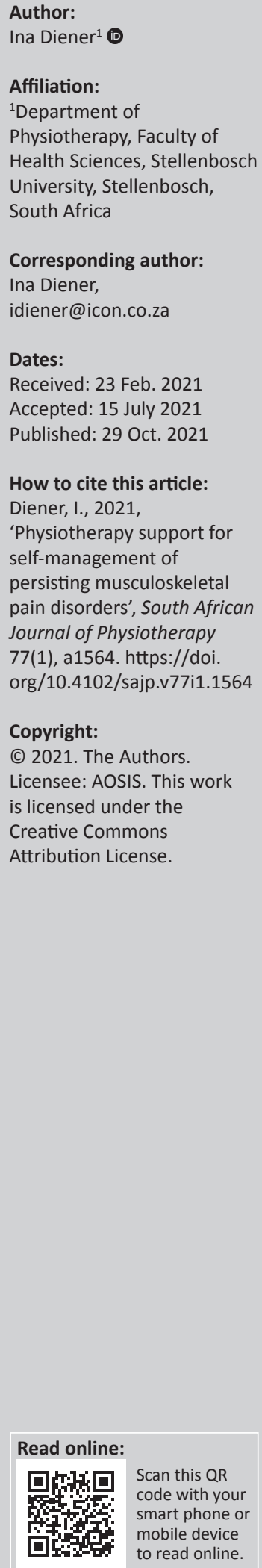

Background: Musculoskeletal pain (MSKP) is an extremely common pain disorder in almost all populations. Self-management (SM) support is a programme to prepare people to selfmanage their health condition effectively, while maintaining quality of life. SM is a costeffective and context-specific strategy to address the global public health burden.

Objectives: Self-management needs a change in behaviour from seeking unnecessary medical care to safely self-managing symptoms. As changing individuals' behaviour is challenging, the objective of my literature review was to identify the characteristics, in both therapist and patient, to successfully engage in SM.

Method: A narrative literature review, that could inform evidence-based support programmes for SM of MSKP.

Results: Studies on successful implementation of SM of MSKP do not report strong outcomes. However, in more recent years a few positive outcomes were reported, possibly as a result of research evidence for the application of psychosocial skills and contemporary pain neuroscience in the management of persistent MSKP.

Conclusion: Psychologically-informed physiotherapy, addressing psychosocial barriers to the maintenance of SM programmes, could facilitate more successful outcomes.

Clinical implications: Before engaging in a SM support programme, obstacles to behaviour change must be identified and addressed in a SM support programme, to facilitate individuals towards taking safe responsibility for their healthcare. Therapists working with patients with persistent MSKP, should upskill themselves to be in line with the latest pain and psychosocial research literature. Moreover, communication skills training seems to be a priority for effective SM support programmes.

Keywords: self-management; self-management support; musculoskeletal pain; psychosocial barriers; behaviour change; psychologically informed physiotherapy.

\section{Increasing cost and rising disability}

Chronic musculoskeletal pain (MSKP) is a prevalent pain disorder in almost all populations, in low- and high-income countries, and is a leading cause of disability worldwide. Therefore, a narrative literature review was done to inform the introduction of self-management (SM) in healthcare for chronic MSKP. The Global Burden of Disease study in 2019 demonstrated that musculoskeletal disorders are amongst the 10 most important drivers of increasing burden, and globally there is growing disability as a result of MSKP conditions. The highest contribution to the need for rehabilitation comes from musculoskeletal disorders. Low back pain was the leading health condition contributing to the need for rehabilitation services in 134 out of the 204 countries analysed; and it is growing rapidly in low- and middle-income countries (Cieza et al. 2020). Epidemiological studies have highlighted that psychosocial factors are associated with MSKP and disability, and have informed us on the way in which these factors serve as prognostic indicators for the development of chronic pain. Findings indicate that several dimensions of patients' illness perceptions are key predictors of pain, and are significantly associated with a more severe pain trajectory years later (Foster \& Delitto 2011). Understanding the long-term trajectories of MSKP disorders should enable better management of the long-term course of persistent MSKP, and could form part of patient education for SM interventions.

Recent research outcomes demonstrate the need for a shift towards a biopsychosocial focus in healthcare (i.e. cognition and perceptions, and social context) which underlies the pain problem, 
rather than only focusing on a purely biomedical origin when treating patients with persistent MSKP (Foster \& Delitto 2011; Lin et al. 2020). Persistent pain is a complex disorder where central and peripheral nociceptive processes are influenced by factors from multiple dimensions. The recent developments in pain science have changed the role of physiotherapy in MSKP disorders. Research on pain has largely converged in support of three concepts that have shifted the healthcare profession's understanding and treatment of pain. Firstly, that pain is not a signal originating from bodily tissues; secondly, that pain is not an accurate measure of tissue injury; and thirdly, that the nervous system demonstrates plasticity, which then becomes a viable target of treatment (Parker \& Madden 2020). Consistent recommendations for the management of persistent MSKP include, amongst others: patient-centred care; red flag and psychosocial factor screening; minimum radiological imaging; thorough first clinical examination; patient education; assessment and address of functional physical activity; and facilitating continuation or resumption of work (Lin et al. 2020).

Persistent MSKP has huge negative effects on people's wellbeing and cost to society. Treatment outcomes for people with persistent MSKP are modest and related healthcare cost is high. Healthcare systems globally are facing great challenges being increasingly under pressure to provide financially unsustainable healthcare services to patients with persistent MSKP (Cieza et al. 2020; Foster \& Delitto 2011). In considering how best to develop an effective system that delivers quality care and value for money, the role that patients play has become ever more important. Selfmanagement is a safe, community-based and effective way for patients with persistent MSKP to manage pain and disability. Self-management support involves provision of interventions to prepare people to manage health conditions effectively and maintain quality of life, and should therefore identify obstacles to change of behaviour. It should also support individuals towards safely taking responsibility for their own healthcare.

\section{Self-management: The current state of evidence}

Self-management is endorsed in most guidelines for the management of MSKP, but the outcomes from studies on SM are not promising. Several systematic literature reviews demonstrate that SM has no or marginal benefit for patients with MSKP disorders (Elbers et al. 2018; Oliveira et al. 2012a; Reid et al. 2008). A few systematic reviews on the effectiveness of SM suggest improvements in knowledge and self-efficacy, but small or negligible effects on function in comparison to usual care, wait-list, or attention-controls (Du et al. 2011; Kroon et al. 2014; Nolte \& Osborne 2013). In an analysis of the results of several randomised controlled trials (RCTs), strong evidence was found that self-efficacy and depression at baseline predict outcome, and that pain catastrophising and physical activity can mediate the outcome of SM (Miles et al.
2011). Other potential explanations for weak results in SM studies mentioned by these authors include: sub-grouping of patients with particular characteristics may be more effective than generic SM programmes; a strong therapeutic relationship with the participant could facilitate a change in behaviour towards SM. It has, however, been demonstrated that lasting behaviour change is challenging (Webb \& Sheeran 2006). Self-management support programmes should be designed to take these factors into account.

Recently, support programmes for SM have been developed to incorporate contemporary pain science and the recent evidence on how to best manage chronic MSKP with a combination of education and exercise. Some noteworthy examples are from Denmark, where a group of researchers formulated the 'Good Life with Osteoarthritis (OA) in Denmark' (GLA:D ${ }^{\mathrm{TM}}$ ) programme for SM support for people with knee and hip OA (Skou \& Roos 2017). The GLA:D ${ }^{\mathrm{TM}}$ programme for patients comprises a 'minimal intervention' with three sessions of patient education delivered over 2 weeks and 12 sessions of supervised neuromuscular exercise delivered twice weekly for 6 weeks. The patient education emphasised knowledge of OA and treatment of OA with a particular focus on exercise, its beneficial effects on symptoms and general health, and self-help advice. After the 8-week programme, the patients are encouraged to continue being physically active and to exercise. Individual strategies for the continuation of physical activity and exercise are discussed at the 3-month follow-up. After having participated in the GLA:D Kn Knee and Hip' programmes, patients' pain decreased by $26 \%-27 \%$, function improved, fewer people took pain-killers and fewer people were on sick leave. The same group of researchers have now developed an evidencebased SM support programme for chronic low back pain, with the aim to improve pain beliefs and management skills (Kjaer et al. 2018). Patient education is provided in two 1-h lectures, and includes pain mechanisms, active coping strategies, imaging, physical activity and exercise. The exercise programme includes 16 supervised 1-h sessions over 8 weeks, with the aim to improve overall back fitness and, at the same time, encourage patients to explore variations in movement by incorporating education content into the exercise sessions. A pilot study demonstrated the programme feasibility for a nation-wide RCT, and secondary outcomes demonstrated better patient-reported outcomes compared to before the programme was implemented.

A 6-week 'ChrOnic pain self-ManageMent with pain science EducatioN and exerCisE' (COMMENCE) programme, resulted in improved function, pain intensity, pain knowledge, catastrophising, self-efficacy, satisfaction with health care, and increased 'global rating of change' scores over a 12-week follow-up period in comparison with usual care (Miller et al. 2020). The SM support programme included education about SM, pain science and cognitive behavioural principles to support behaviour change, combined with an exercise programme tailored to participants' goals and abilities. The SM support programme led by trained 
physiotherapists, consisted of two visits per week over 6 weeks. One visit in a group format incorporated education about SM and pain science, and cognitive behavioural principles to support behaviour change. The second was individually tailored, aiming to support implementation of SM plans and the development of an exercise programme adapted to the participant's goals and abilities. Participants were encouraged to continue SM beyond the end of the intervention up to measurement at 12 weeks follow-up. The COMMENCE programme was more effective than usual care at improving function, pain, catastrophic thinking, selfefficacy, pain knowledge, satisfaction, and perceived change, in a group of patients with chronic non-cancer pain.

It can be challenging to effectively integrate behavioural and cognitive components into the biopsychosocial management of MSKP. Depression and self-efficacy predicted outcome irrespective of intervention, suggesting that these should be targeted at early stages to prevent the transition to chronic disability (Miles et al. 2011; Webb \& Sheeran 2006). This was confirmed by a recent literature review of the intervention components and patient outcomes of studies integrating these components in physiotherapy. The researchers' aim was to match the interventions with a definition of behavioural medicine in physiotherapy, while categorising the behaviour change techniques used for patients with MSKP. The results demonstrated that the integrated psychosocial, behavioural, and biomedical or physical aspects applied in the reviewed interventions complied with the existing definition of behavioural medicine in physiotherapy. The frequently reported component was to improve self-efficacy. The long-term follow-ups mostly showed positive effects, for example, reduction of fear and catastrophising, change in pain beliefs, increase in activities and pain SM strategies, and improved stress management (Soderlund et al. 2020). The findings of the study suggest that factors like self-efficacy, depression, pain catastrophising and physical activity impact on outcomes of the SM programmes. There is evidence that understanding of pain neuroscience and early identification of psychological barriers, may improve these factors (Diener, Kargela \& Louw 2016; Louw et al. 2016). Utilising these in the SM support programmes may thus improve the outcomes of these programmes.

\section{Support programmes to prepare individuals with persistent musculoskeletal pain for self- management}

The SM support should prepare people to manage health conditions effectively and maintain quality of life. The preparatory programme should thus prepare and empower patients towards SM of their chronic MSKP disorders, and support them during the process (Cooper, Smith \& Hancock 2009; Hutting et al. 2020; Peek et al. 2016). Psychological adjustment in resilience and preparedness to take responsibility may lead to favourable outcomes. For successful SM, a person with persistent MSKP must be able to manage the symptoms, treatment, physical and psychosocial consequences, and lifestyle changes inherent to living with a chronic condition. Ideally, a SM support programme should address all these factors, as newly learned behaviours can be difficult to maintain.

The outcome of a support programme for SM should be measured in terms of 'enablement' of the patient. As 'enablement' can mirror the results differently from traditionally used outcome measures, outcomes should be measured with questions such as: whether the programme has been successful in empowering and supporting patients to have strategies to regain power over their own lives, accept their current status, achieve better health and reach their personal goals. The Patient Enablement Instrument (PEI) has been found to have fair content validity, construct validity and internal consistency in a group of patients with chronic MSKP (Enthoven et al. 2019).

\section{What is likely needed for successful self-management of a musculoskeletal pain disorder?}

\section{Tutors of self-management support programmes}

Self-management and SM support are considered important to physiotherapists, for people with MSKP disorders, but the way physiotherapists address this in practice does not seem to be optimal (Miles et al. 2011). Many clinicians hold unhelpful beliefs themselves; while others feel ill-equipped to explore and target the patient's unhelpful beliefs that drive the pain. As a result, clinicians may reinforce unhelpful beliefs, behaviours and resultant disability among the patients they train for SM (Caneiro, Bunzli \& O'Sullivan 2020). Elbers et al. (2018) suggested three reasons for SM programmes not being successful: behavioural change is difficult; self-efficacy for SM is often lacking; and empowerment towards active and resilient coping needs to be included. Patients express a need for SM support, suggesting there is room for empowerment towards selfmanaging their condition (Cooper et al. 2009).

Effective implementation of SM support programmes thus depends partially on the skills of service providers, of which communication skills seem to be the most important, to understand the individual and the barriers that may be faced when embarking on SM of persistent MSKP. Selfmanagement interventions need tailoring to individual barriers, facilitators and needs as these play an important role in realising positive effects. Healthcare providers, may require additional training on how to collaborate with their patients or clients in setting health goals and decisionmaking (Mann, LeFort \& VanDenKerkhof 2013). Experts in the field identified four factors that should guide the SM support provided: change of mind-set; a patient-specific programme; managing uncertainty; and support from the therapist (Stenner et al. 2015). 
Physiotherapists are in a good position to empower patients towards SM of MSKP, and are frequently considered as part of the rehabilitation team (Hutting et al. 2020; Soderlund et al. 2020). Results from some successful outcomes in studies on the management of persistent MSKP have informed us on what kind of therapy or therapist is needed in the support programme, for SM to be successful (Foster \& Delitto 2011). Therapists' own beliefs about MSKP and the best management thereof plays an important role in the way a SM support programme is structured (Caneiro et al. 2020). Healthcare providers therefore need to have knowledge of the current evidence-base of care for persistent pain (Lin et al 2020; Parker \& Madden 2020). Also, they should strive to follow researched requirements to implement a successful SM support programme and facilitate adherence to the programme (Hutting et al. 2020; Peek et al. 2016:127-135). A holistic patient-centred approach by the healthcare provider is one comprised of the three roles of: educator, partner and coach (Solvang \& Fougner 2016:591-602), suggesting that changing to $\mathrm{SM}$, needs education of the patient in a strong therapeutic relationship, and awareness of the living conditions of the patient. To fulfil these three roles, a therapist involved in the preparatory support programme, needs certain attributes to facilitate a successful SM outcome.

\section{Communication skills}

Clinical communication skills and a strong therapeutic relationship may facilitate person-centred care, shared decision-making and goal setting. Listening and motivating clinical communication, as meaningful conversations, may encourage an active role for people under therapists' care. Listening is therapy in itself; it demonstrates to the person that their 'story' is valuable (Diener et al. 2016). Understanding what is important to a person and insights into a person's life is vital to ensure a positive outcome in SM. Exploring the psychosocial context of the person in pain includes their expectations, existing depression and anxiety factors, and their social support system (Miller et al. 2020). Good communication between health providers and patients, and valuing patient autonomy can promote adherence and resultantly improve outcomes (Oliveira et al. 2012b). Motivational interviewing (MI) aims to strengthen personal commitment by respecting the individual's autonomy and assisting them to reach a specific goal by exploring personal intentions or reasons for change (Alperstein \& Sharpe 2016). Motivational interviewing is valuable to facilitate peoples' desire to help themselves and to facilitate a behavioural change. Patient education and communication strategies such as pain neuroscience education and MI have been developed for the management of persistent disabling pain. (Nijs et al. 2020). Training healthcare providers in clinical communication skills can enhance patient outcomes; and this needs to be a priority for the providers of the SM support programmes.

A strong therapeutic relationship is built on trust and mutual respect and is dependent on effective clinical communication skills. In a qualitative study, participants made it clear that therapeutic relationships do not 'just happen'. Clinicians need to be present (in-the-moment), receptive (an open attitude and a focused receptivity), genuine (being yourself, and being honest), and committed (be engaged in your role). The therapeutic relationship between patient and provider is considered a central component of patient-centred care and patient engagement, leading to patient satisfaction and adherence to treatment (Miciak et al. 2018). Patient-centred care emphasises equal partnerships between people involved in planning, developing and accessing care to ensure it meets the person's needs. An overwhelming amount of evidence supporting a person-centred approach has placed it at the core of healthcare for people living with long term conditions (Cooper et al. 2009; Lin et al. 2020; Miciak et al. 2018; Nijs et al. 2020; Peek et al. 2016). Clinicians need to have skills and knowledge in both evidence-based practice and communication, so that both evidence and patient preferences can be incorporated into consultations (Nijs et al. 2020).

\section{Educational skills}

Firstly, clinicians' awareness and knowledge of psychosocial contributions to persistent pain is important for support and education of the patient. Screening for recommended psychosocial risk factors (aka yellow flags), which may be barriers to recovery has been researched abundantly in recent years. It has become apparent that these psychosocial risk factors, like fear of movement, anxiety, maladaptive beliefs, etc., may in many cases be the dominating factors associated with recovery in MSKP disorders, and need to be identified and addressed (Diener et al. 2016; Foster \& Delitto 2011; Lin et al. 2020; Parker \& Madden 2020). The role of vulnerability factors such as pain-related fear, catastrophising, and avoidance have been well-documented in the development and maintenance of chronic pain disability (Meulders 2019). Caneiro et al. (2020) encouraged clinicians to exercise selfreflection to explore their own beliefs and better understand their biases, which may influence their management of patients with MSKP. They are of the opinion that disconfirming unhelpful beliefs through behavioural learning, self-reflection and evidence-based education, can promote a new understanding that empowers SM.

Secondly, health literacy should form part of education of the patient. Health literacy describes the personal skills and environmental conditions that enable individuals to obtain, understand, and use information to make decisions and take actions that will have an impact on their health status (Muscat et al. 2020). Being knowledgeable about health issues could facilitate shared decision-making. Even though shared decision-making has not been tested for its role in the outcome of management of chronic MSKP conditions, there is a potential benefit in a patient-centred care approach, as it gives a voice to individuals and allows them more control towards the healthcare they choose to receive, and their own participation (Tousignant-Laflamme et al. 2017). Health education supports the patient's thought processes and decision-making. This information should, however, always follow the principles of contemporary pain neuroscience, to not increase pain related fear (Louw et al. 2016). Physiotherapists providing care for people presenting with 
musculoskeletal conditions are in the privileged position of being able to promote healthy behaviour (Lewis et al. 2021) by providing evidence-based education on:

- the natural course of MSKP conditions (Foster \& Delitto 2011; Lin et al. 2020; Parker \& Madden 2020)

- the evidence-base for radiological imaging and the negative effect of unnecessary scanning (Lin et al. 2020; Caneiro et al. 2020)

- the benefits of activity and exercise (Elbers et al. 2018; Kjaer et al. 2018; Lin et al. 2020; Miller et al. 2020; Skou \& Roos 2017; Wade 2020).

The third part of education is therapeutic neuroscience education (TNE), that has been demonstrated to help patients to reconceptualise their pain, leading to reduction in pain, disability, psychosocial factors and healthcare utilisation, while improving movement and function (Louw et al. 2016). The aim of the TNE is to guide patients' pain beliefs and perceptions to facilitate the acquisition of adaptive paincoping strategies (Diener et al. 2016; Nijs et al. 2020).

\section{Physical examination and clinical reasoning skills}

The clinician, embarking on a SM support programme for a patient, should have the knowledge and skill to do an appropriate musculoskeletal physical examination, to rule out red flags and confirm the pain type the patient is complaining of. Clinical reasoning based on a thorough interview and physical examination should differentiate nociceptive versus neuropathic versus nociplastic pain, which each needs a specific SM support approach. Whereas nociceptive and neuropathic pain may need advice on positions and movements, SM support for nociplastic pain should care for the psychosocial factors driving the pain experience. Pain should never be accepted as persistent pain with no apparent physical reason before a thorough questioning and physical examination have cleared all red flags and confirmed the type of pain.

\section{Physical rehabilitation skills}

Rehabilitation is about helping people recover from illness to return to what matters in life. The end-goal of rehabilitation is to optimise a patient's self-rated quality of life and degree of social integration through optimising independence in activities, minimising pain and distress, and optimising the ability to adapt and respond to changes in circumstances (Wade 2020). Effective rehabilitation is a person-centred process, tailored to the individual patient's needs, and importantly, personalised monitoring of changes associated with intervention. However, patients are often not adherent to rehabilitation interventions, and non-adherence can compromise the gains seen from an effective SM support programme. A strong therapeutic relationship, shared decision-making and patient-centred clinical communication may enhance adherence and patient outcomes. The SM support programme must therefore, for each individual patient, use a collection of interventions to meet the patient's specific needs and evaluate the patient's rehabilitation programme on a planned, ongoing basis, using simple targeted
TABLE 1: Attributes of therapists supporting patients with musculoskeletal pain disorders towards SM.

\begin{tabular}{|c|c|c|}
\hline $\begin{array}{l}\text { Attributes of the } \\
\text { therapist engaging in a } \\
\text { SM support programme }\end{array}$ & Components & Reading \\
\hline Communication skills & $\begin{array}{l}\text { Listening and reflecting } \\
\text { Therapeutic alliance } \\
\text { Motivational interviewing }\end{array}$ & $\begin{array}{l}\text { Diener et al. } \\
\text { (2016); Miciak } \\
\text { et al. (2018); Nijs } \\
\text { et al. (2020) }\end{array}$ \\
\hline Educational skills & $\begin{array}{l}\text { Addressing psychosocial barriers to } \\
\text { engaging in a SM programme } \\
\text { Self-reflection on own beliefs } \\
\text { Health education } \\
\text { Pain neuroscience education }\end{array}$ & $\begin{array}{l}\text { Meulders (2019); } \\
\text { Caneiro et al. } \\
\text { (2020) }\end{array}$ \\
\hline $\begin{array}{l}\text { Physical rehabilitation } \\
\text { skills }\end{array}$ & $\begin{array}{l}\text { Person-centred } \\
\text { Needs-tailored } \\
\text { Aspects to improve adherence }\end{array}$ & Wade (2020) \\
\hline
\end{tabular}

SM, self-management.

measures at appropriate intervals. By promoting behavioural change (Katz, Patterson \& Zacharias 2019) and empowering participants to take control (Zuercher-Huerlimann et al. 2019), rehabilitation programmes may be maintained by participants. Physiotherapists need to ask themselves if they understand the importance of behavioural change and if they have the skills and knowledge to incorporate behavioural change as an integral part of the rehabilitation they offer (Lewis et al. 2021). Table 1 is a summary of the attributes therapists engaging in SM programmes should strive to master.

\section{Participants of self-management programmes}

In preparing for $\mathrm{SM}$, clinicians should focus on important SM skills that participants possess. It is therefore important during the support programme, to assess participants' skills and knowledge of SM, and their confidence in managing their own health. Factors such as readiness for change, health locus of control (HLOC), patient activation level of health, self-efficacy and pain beliefs need to be assessed and addressed during the support programme with training, to facilitate a successful transition to SM (Martin et al. 2019). Showing willingness to engage in SM of their persisting MSKP is in principle a mind-set and behaviour change. Therefore, behavioural counselling may help to increase their adherence to the programme, and boosting self-efficacy may facilitate a positive lifestyle change (Lewis et al. 2021). The SM support programmes should include assessment and training of the skills required for day-to-day management of chronic pain conditions, to ensure that individual barriers to SM are overcome and it succeeds.

\section{Readiness for behaviour change}

Patients included in a SM programme before they are ready to take responsibility, may fail to change their behaviour and thus not succeed The study of Katz et al. (2019) demonstrated the importance of a potential pre-programme targeted intervention for better patient engagement in an interdisciplinary pain programme. The 'Pain Stages of Change' (pre-contemplation, contemplation, action, and maintenance) Questionnaire is a useful tool to help identify how ready a patient is for behaviour change towards SM of their MSKP. 


\section{Health locus of control}

The need for SM is individual-specific and may change over time. The HLOC refers to the extent to which individuals believe they can control events affecting their MSKP and other health conditions (Grotz et al. 2011) A person's 'locus' is conceptualised as either internal or external. The internal locus refers to the extent to which a person believes they can influence their own life situation. An external locus refers to their belief that their decisions and life are beyond their own influence, decided by environmental factors which they cannot influence, or that decisions and life are determined by chance or fate. Higher levels of internal control are related to better SM competency (Zuercher-Huerlimann et al. 2019). The HLOC needs to be addressed in a preparatory programme for $\mathrm{SM}$, to build confidence in the ability to exert control over motivation and behaviour.

\section{Pain beliefs and self-efficacy}

There are several maladaptive beliefs among individuals with a persistent MSKP. An 8-week interdisciplinary pain programme, associated with improvements in patientrelated outcomes, demonstrated that fear of pain or re-injury was predictive of a patient being in the pre-contemplative stage of change, whereas pain self-efficacy was predictive of being in the contemplative, action, or maintenance stage of change (Katz et al. 2019). Illness beliefs, fear, expectation and perception of physical capacity influence self-efficacy (Caniero et al. 2020). Fear of movement is one of the most important reasons for the development of chronic painrelated disability (Meulders 2019). It is therefore important to have targeted interventions to decrease fear of pain or reinjury, and to enhance self-efficacy for better patient engagement and compliance. Believing that an injury or dysfunction is the reason for a patient's pain and fear of movement-related pain, should be addressed promptly, as this can delay patients taking responsibility for SM. Radiological imaging results, biomedical explanations for their pain by medical practitioners, and internet information may all fuel maladaptive fear of pain or re-injury (Caneiro et al. 2020; Lin et al. 2020). Fearful participants may also need more support after they have started with SM, as flair-up episodes of pain may let them sink back into their mal-beliefs. Table 2 is a summary of the attributes participants engaging in SM programmes need.

TABLE 2: Attributes that participants most likely need to engage in successful self-management.

\begin{tabular}{lll}
\hline $\begin{array}{l}\text { Attributes of the } \\
\text { patient engaging in a } \\
\text { SM support programme }\end{array}$ & Assessment & Reading \\
\hline $\begin{array}{l}\text { Readiness for } \\
\text { behaviour change }\end{array}$ & $\begin{array}{l}\text { Pain Stages of Change } \\
\text { Questionnaire }\end{array}$ & Kerns (1997) \\
HLOC & HLOC & $\begin{array}{l}\text { Wallston, Kaplan and } \\
\text { Maides (1976) }\end{array}$ \\
$\begin{array}{lll}\text { Pain beliefs and } \\
\text { self-efficacy }\end{array}$ & $\begin{array}{l}\text { Pain Catastrophising Scale } \\
\text { Tampa Scale for } \\
\text { Kinesiophobiae11 } \\
\text { Pain Self-Efficacy Questionnaire } \\
\text { Patient Health Questionnaire }\end{array}$ & $\begin{array}{l}\text { Sullivan, Bishop and Pivik } \\
\text { (1995); Tkachuk and } \\
\text { (2007); Kroenke, Spitzer } \\
\text { and Williams (2001) }\end{array}$ \\
\hline
\end{tabular}

SM, self-management; HLOC, Health locus of control

\section{Conclusion}

Epidemiological research demonstrates that persistent MSKP disorders pose a significant worldwide epidemiologic burden, displaying an escalating trend. There is an increasing recognition of the merits of 'de-medicalising' musculoskeletal care and shifting towards models of care that are more patientcentred and focused on supporting SM. The SM support programmes should in essence prepare participants for behaviour change, but should also be in place to provide support during the SM period. Self-management, a common component in interdisciplinary pain management programmes, is expected to facilitate more active and resilient coping. Healthcare providers working with patients with persistent MSKP should upskill to be in line with the latest pain neurophysiology and pain-related psychosocial research literature. Moreover, communication skills training of therapists needs to be a priority for effective SM support programmes. Research into SM support programmes for MSKP not only need to include the best treatment and rehabilitation techniques, but also assessment and training of psychosocial components that will facilitate and support behaviour change and compliance to SM. Although there are several global initiatives to address the global burden of persistent pain as a public health problem, there is a need to identify cost-effective and context-specific strategies for managing persistent MSKP disorders, to reduce the consequences of the current burden. Self-management support programmes are seen as one of the possibilities. Clinicians have a collective responsibility to educate patients, the community, funders, policymakers and other clinicians on safe and effective SM of MSKP, to help reduce the disability and cost burden in society, especially in a country like South Africa, where the disability burden is high and health resources are limited.

\section{Acknowledgements Competing interests}

The author declares that they she has no financial or personal relationships that may have inappropriately influenced her in writing this article.

\section{Author's contributions}

I.D. declares that she is the sole author of this research article.

\section{Ethical considerations}

This article followed all ethical standards for research without direct contact with human or animal subjects.

\section{Funding information}

No financial support was received for the research, authorship and/or publication of this article.

\section{Data availability}

Data sharing is not applicable to this article as no new data were created or analysed in this study. 


\section{Disclaimer}

The views and opinions expressed in this article are those of the author and do not necessarily reflect the official policy or position of any affiliated agency of the author.

\section{References}

Alperstein, D. \& Sharpe, L., 2016, 'The efficacy of motivational interviewing in adults with chronic pain: A meta-analysis and systematic review', The Journal of Pain 17(4), 393-403. https://doi.org/10.1016/j.jpain.2015.10.021

Caneiro, J.P., Bunzli, S. \& O'Sullivan, P.B., 2020, 'Beliefs about the body and pain: The critical role in musculoskeletal pain management', Brazilian Journal Physical critical role in musculoskeletal pain management', Brazilian Journ
Therapy 25(1), 17-29. https://doi.org/10.1016/j.bjpt.2020.06.003

Cieza, A., Causey, K., Kamenov, K., Hanson, S.W., Chatterji, S. \& Vos, T., 2020, 'Global estimates of the need for rehabilitation based on the Global Burden of Disease
study 2019: A systematic analysis for the Global Burden of Disease Study 2019', The Lancet 396(10267), 2006-2017. https://doi.org/10.1016/S0140-6736(20)32340-0 Cooper, K., Smith, B.H. \& Hancock, E., 2009, 'Patients' perceptions of self-management
of chronic low back pain: Evidence for enhancing patient education and support', Physiotherapy 95, 43-50. https://doi.org/10.1016/j.physio.2008.08.005

Diener, I., Kargela, M. \& Louw, A., 2016, 'Listening is therapy: Patient interviewing from a pain science perspective', Physiotherapy Theory and Practice 32(5), 356-367. https://doi.org/10.1080/09593985.2016.1194648

Du, S., Yuan, C., Xiao, X., Chu, J., Qiu, Y. \& Qian, H., 2011, 'Self-management programs for chronic musculoskeletal pain conditions: A systematic review and metaanalysis', Patient Education and Counselling 85(3), e299-310. https://doi org/10.1016/j.pec.2011.02.021

Enthoven, P., Peolsson, A., Landén Ludvigsson, M., Wibault, J., Peterson, G. \& Öberg, B. 2019, 'Validity, internal consistency and self-rated change of the patient enablement instrument in patients with chronic musculoskeletal pain', Journal of
Rehabilitation Medicine 51(8), 587-597. https://doi.org/10.2340/16501977-2573

Elbers, S., Wittink H., Pool, J.J. \& Smeets, R.J., 2018, 'The effectiveness of generic selfmanagement interventions for patients with chronic musculoskeletal pain on physical function, self-efficacy, pain intensity and physical activity: A systematic review and meta-analysis', European Journal of Pain 22(9), 1577-1596. https:// doi.org/10.1002/ejp.1253

Foster, N.E. \& Delitto, A., 2011, 'Embedding psychosocial perspectives within clinical management of low back pain: Integration of psychosocially informed management principles into physical therapist practice - Challenges and opportunities', Physical Therapy 91(5), 790-803. https://doi.org/10.2522/ptj.20100326

Grotz, M., Hapke, U., Lampert, T. \& Baumeister, H., 2011, 'Health locus of contro and health behaviour: Results from a nationally representative survey', Psychology, Health \& Medicine 16(2), 129-140. https://doi.org/10.1080/13548 506.2010.521570

Hutting, N., Oswald, W., Staal, J.B. \& Heerkens, Y.F., 2020, 'Self-management support for people with non-specific low back pain: A qualitative survey among physiotherapists and exercise therapists', Musculoskeletal Science and Practice 50, 102269. https://doi.org/10.1016/j.msksp.2020.102269

Katz, L., Patterson, L. \& Zacharias, R., 2019, 'Evaluation of an interdisciplinary chronic pain program and predictors of readiness for change', Canadian Journal of Pain pain program and predictors of readiness for change', Canadion
$3(1), 70-78$. https://doi.org/10.1080/24740527.2019.1582296

Kerns, R.D., Rosenberg, R., Jamison, R.N., Caudill, M.A. \& Haythornthwaite, J., 1997, 'Readiness to adopt a self-management approach to chronic pain: The Pain Stages of Change Questionnaire (PSOCQ)', Pain 72(1), 227-234. https://doi.org/10.1016/ S0304-3959(97)00038-9

Kjaer, P., Kongsted, A., Ris, I., Abbott, A., Rasmussen, C.D., Roos, E.M. et al., 2018 'GLA: $D^{\circledast}$ back group-based patient education integrated with exercises to support self-management of back pain-development, theories and scientific evidence', BMC
Musculoskeletal Disorders 19(1), 418. https://doi.org/10.1186/s12891-018-2334-x

Kroenke, K. \& Spitzer, R.L. \& Williams, J.B., 2001, 'The PHQ-9', Journal of General Internal Medicine 16, 606-613. https://doi.org/10.1046/j.1525-1497.2001.016009606.x

Kroon, F.P.B., Van der Burg, L.R.A., Buchbinder, R., Osborne, R.H., Johnston, R.V. \& Pitt, V., 2014, 'Self-management education programmes for osteoarthritis', Cochrane Database of Systematic Reviews 2014(1), CD008963. https://doi. org/10.1002/14651858.CD008963.pub2

Lewis, J., Ridehalgh, C., Moore, A. \& Hall, K., 2021, 'This is the day your life must surely change. Prioritising behavioural change in musculoskeletal practice', Physiotherapy 112, 158-162. https://doi.org/10.1016/j.physio.2021.05.007

Lin, I., Wiles, L., Waller, R., Goucke, R., Nagree, Y., Gibberd, M. et al., 2020, 'What does best practice care for musculoskeletal pain look like? Eleven consistent recommendations from high-quality clinical practice guidelines: Systematic review', British Journal of Sports Medicine 54(2), 79-86. https://doi.org/10.1136/ bjsports-2018-099878

Louw, A., Zimney, K., Puentedura, E.J. \& Diener, I., 2016, 'The efficacy of pain neuroscience education on musculoskeletal pain: A systematic review of the literature', Physiotherapy Theory and Practice 32(5), 332-355. https://doi.org/10. 1080/09593985.2016.1194646

Mann, E.G., LeFort, S. \& VanDenKerkhof, E.G., 2013, 'Self-management interventions fo chronic pain', Pain Management 3(3), 211-222. https://doi.org/10.2217/pmt.13.9

Martin, E.S., Dobson, F., Hall, M., Marshall, C. \& Egerton, T., 2019, 'The effects of behavioural counselling on the determinants of health behaviour change in adults with chronic musculoskeletal conditions making lifestyle changes: A systematic review and meta-analysis', Musculoskeletal Care 17(3), 170-197. https://doi. org/10.1002/msc.1410
Meulders, A., 2019, 'From fear of movement-related pain and avoidance to chronic pain disability: A state-of-the-art review', Current Opinion in Behavioral Sciences pain disability: A state-of-the-art review', Current Opinion in

Miciak, M., Mayan, M., Brown, C., Joyce A.S. \& Gross, D.P., 2018, 'The necessary conditions of engagement for the therapeutic relationship in physiotherapy: An interpretive description study', Archives of Physiotherapy 8(3), 1-12. https://doi. org/10.1186/s40945-018-0044-1

Miles, C.L., Pincus, T., Carnes, D., Homer, K.H., Taylor, S.J.C., Bremner, S.A. et al., 2011 'Can we identify how programmes aimed at promoting self-management in musculoskeletal pain work and who benefits? A systematic review of sub-group analysis within RCTs', European Journal of Pain 15(8), 775. https://doi. org/10.1016/j.ejpain.2011.01.016

Miller, J., MacDermid, J.C., Walton, D.M. \& Richardson, J., 2020, 'Chronic pain selfmanagement support with pain science education and exercise (COMMENCE) for people with chronic pain and multiple comorbidities: A randomized controlled trial', Archives of Physical Medicine and Rehabilitation 101(5), 750-761. https:// doi.org/10.1016/j.apmr.2019.12.016

Muscat, D.M., Shepherd, H.L., Nutbeam, D., Trevena, L. \& McCaffery, K.J., 2020 'Health literacy and shared decision-making: Exploring the relationship to enable 'Healthingful patient engagement in healthcare', Journal of General Internal Medicine 36, 521-524. https://doi.org/10.1007/s11606-020-05912-0

Nicholas, M.K., 2007, 'The pain self-efficacy questionnaire: Taking pain into account', European Journal of Pain 11(2), 153-163. https://doi.org/10.1016/j. ejpain.2005.12.008

Nijs, J., Wijma, A.J., Willaert, W., Huysman, S.E., Mintken, P., Smeets, R. et al., 2020 Integrating motivational interviewing in pain neuroscience education for people with chronic pain: A practical guide for clinicians', Physical Therapy 100(5), 846-859. https://doi.org/10.1093/ptj/pzaa021

Nolte, S. \& Osborne, R.H., 2013, 'A systematic review of outcomes of chronic disease self-management interventions', Quality of Life Research 22, 1805-1816. https:// doi.org/10.1007/s11136-012-0302-8

Oliveira, V.C., Ferreira, P.H., Maher, C.G., Pinto, R.Z, Kathryn M., Refshauge, K.M. et al, 2012a, 'Effectiveness of self-management of low back pain: Systematic review $2012 a$, 'Effectiveness of self-management of low back pain: Systematic review
with meta-analysis', Arthritis Care \& Research 64(11), 1739-1748. https://doi. with meta-analysis', Arthr
org/10.1002/acr.21737

Oliveira, V.C., Refshauge, K.M., Ferreira, M.L., Pinto, R.Z., Beckenkamp, P.R., Negrao Filho, R.F. et al., 2012b, 'Communication that values patient autonomy is associated with satisfaction with care: A systematic review', Journal of Physiotherapy 58(4), 215-229. https://doi.org/10.1016/S1836-9553(12)70123-6

Parker, R. \& Madden, V.J., 2020, 'State of the art: What have the pain sciences brought to physiotherapy?', South African Journal of Physiotherapy 76(1), a1390. https:// doi.org/10.4102/sajp.v76i1.1390

Peek, K., Sanson-Fisher, R., Mackenzie, L. \& Carey, M., 2016, 'Interventions to aid patient adherence to physiotherapist prescribed self-management strategies: A systematic review', Physiotherapy 102(2), 127-135. https://doi.org/10.1016/j. physio.2015.10.003

Reid, M.C., Papaleontiou, M., Ong, A., Breckman, R., Wethington, E. \& Pillemer, K. 2008 , 'Self-management strategies to reduce pain and improve function among 2008, 'Self-management strategies to reduce pain and improve function among older adults in community settings: A review of the evidence',

Skou, S.T. \& Roos, E.M., 2017, 'Good Life with osteoArthritis in Denmark (GLA: D'M): Evidence-based education and supervised neuromuscular exercise delivered by certified physiotherapists nationwide', BMC Musculoskeletal Disorders 18(1), 1-3. https://doi.org/10.1186/s12891-017-1439

Soderlund, A., Elve, M., Sandborgh, M. \& Fritz, J., 2020, 'Implementing a behavioral medicine approach in physiotherapy for patients with musculoskeletal pain: A scopin review', PAIN Reports 5(5), e844. https://doi.org/10.1097/PR9.0000000000000844

Solvang, P.K. \& Fougner, M., 2016, 'Professional roles in physiotherapy practice: educating for self-management, relational matching, and coaching for everyday life', Physiotherapy Theory and Practice 32(8), 591-602. https://doi.org/10.1080/ 09593985.2016.1228018

Stenner, P., Cross, V., McCrum, C., McGowan, J., Defever, E., Lloyd, P. et al., 2015, 'Selfmanagement of chronic low back pain: Four viewpoints from patients and
healthcare providers', Health Psychology Open 2(2), 1-11. https://doi. healthcare providers', Health
org/10.1177/2055102915615337

Sullivan, M.J., Bishop, S. \& Pivik, J., 1995, 'The pain catastrophizing scale: Development and validation', Psychological Assessment 7(4), 432-524. https://doi. org/10.1037/1040-3590.7.4.524

Tkachuk, G.A. \& Harris, C.A., 2012, 'Psychometric properties of the Tampa Scale for Kinesiophobia-11 (TSK-11)', Journal of Pain 13(10), 970-977. https://doi. org/10.1016/j.jpain.2012.07.001

Tousignant-Laflamme, Y., Christopher, C., Clewley, D., Ledbetter, L., Cook C.J. \& Cook, C.E., 2017, 'Does shared decision making results in better health related outcomes for individuals with painful musculoskeletal disorders? A systematic review' Journal of Manual \& Manipulative Therapy 25(30), 144-150. https://doi.org/10.1 080/10669817.2017.1323607

Wade, D., 2020, 'What is rehabilitation? An empirical investigation leading to an evidence-based description', Clinical Rehabilitation 34(5), 571-583. https://doi. evidence-based description', Clinical
org/10.1177/0269215520905112

Wallston, K., Kaplan, G. \& Maides, S., 1976, 'Development and validation of the health locus of control (HLC) scale', Journal of Consulting and Clinical Psychology 44(4), 580-585. https://doi.org/10.1037/0022-006X.44.4.580

Webb, T.L. \& Sheeran, P., 2006, 'Does changing behavioral intentions engender behavior change? A meta-analysis of the experimental evidence', Psychological Bulletin 132(2), 249-268. https://doi.org/10.1037/0033-2909.132.2.249

Zuercher-Huerlimann, E., Stewart, J.A., Egloff, N., Von Känel, R., Studer, M. \& Grosse Holtforth, M., 2019, 'Internal health locus of control as a predictor of pain reduction in multidisciplinary inpatient treatment for chronic pain: A retrospective study', Journal of Pain Research 12, 2095-2099. https://doi.org/10.2147/JPR.S189442 